\title{
CARACTERIZAÇÃO DE CONSTRUÇÕES EM TERRA COMPACTADA UTILIZANDO A PROPAGAÇÃO DE ONDAS ULTRASSÔNICAS
}

\author{
SARRO, WÉLIDA DE SOUSA \\ Doutoranda \\ Faculdade de Tecnologia - UNICAMP \\ São Paulo; Brasil \\ welidasarro@gmail.com
}

\author{
FERREIRA, GISLEIVA C. DOS SANTOS \\ Profa. Dra. \\ Faculdade de Tecnologia - UNICAMP \\ São Paulo; Brasil \\ gisleiva@ft.unicamp.br
}

\section{RESUMO}

\author{
SILVA, TAYNÁ FRACÃO \\ Mestranda \\ Faculdade de Tecnologia - UNICAMP \\ São Paulo; Brasil \\ taynafracao@gmail.com
}

A importância que se dá ao patrimônio histórico e cultural da humanidade passa pela preservação, manutenção e recuperação das construções assim consideradas. Entre as construções identificadas como de interesse histórico, destacam-se aquelas executadas em terra, tanto pela quantidade ainda em serviço como pelo contexto social que estão inseridas. Entretanto, a preservação das construções com terra encontra dificuldades relacionadas às técnicas não destrutivas que podem ser aplicadas neste tipo de material. Para suprir tal problema, há algumas pesquisas sobre a técnica de ultrassom na caracterização de solos. Portanto, o objetivo deste trabalho foi determinar as correlações entre os valores da velocidade do pulso ultrassônico, obtidas com ondas de compressão $\left(\mathrm{V}_{\mathrm{P}}\right)$ e de cisalhamento $\left(\mathrm{V}_{\mathrm{S}}\right)$, e a direção da transmissão do pulso ultrassônico em amostras de solo compactado com diferentes densidades. Foram definidas três direções, em função da metodologia de compactação e posicionamento dos transdutores durante os ensaios de ultrassom: direção de compactação (1) e as faces perpendiculares ao sentido da compactação (2 e 3). A etapa experimental envolveu a confecção de corpos de prova cúbicos (150 mm de aresta) confeccionados com solo argiloso, os quais foram submetidos aos ensaios de ultrassom com transdutores de ondas de compressão (54 kHz) e cisalhamento (40 kHz). Os resultados indicaram que há correlações diretamente proporcionais entre as velocidades $\left(\mathrm{V}_{\mathrm{P}}\right.$ e $\left.\mathrm{V}_{\mathrm{S}}\right)$ e a densidade aparente dos corpos de prova cúbicos de solo argiloso quando os ensaios de ultrassom são realizados com os transdutores posicionados nas faces laterais dos cubos. Entretanto, quando os transdutores são posicionados no topo e base dos cubos (sentido da compactação), a correlação entre a densidade e as velocidades $\left(\mathrm{V}_{\mathrm{P}} \mathrm{e} \mathrm{V}_{\mathrm{S}}\right)$ é inversamente proporcional. Isso pode ser justificado pelo aumento do índice de vazios na região de interface das camadas de compactação, o que dificulta a passagem da onda ultrassônica.

Palavras-chave: taipa de pilão, solo compactado, ensaios não destrutivos, inspeção.

\section{ABSTRACT}

The importance given to the historical and cultural heritage of humanity passes through the preservation, maintenance and restoration of buildings regarded as such. Among the constructions identified as being of historical interest, those built on land stand out, both for the amount still in service and for the social context they are inserted. However, the preservation of earthen constructions faces difficulties related to non-destructive techniques that can be applied to this type of material. To address this problem, there is some research on the ultrasound technique in soil characterization. Therefore, the aim this research was to determine the correlations between the values of ultrasonic pulse velocity, obtained with compression waves $\left(\mathrm{V}_{\mathrm{P}}\right)$ and shear waves $\left(\mathrm{V}_{\mathrm{S}}\right)$, and the direction of ultrasonic pulse transmission in samples of compacted soil with different densities. Three directions were defined, according to the methodology of compaction and positioning of the transducers during the ultrasound tests: direction of compaction (1) and the faces perpendicular to the direction of compaction (2 and 3). The experimental stage involved making cubic specimens (150 mm of edge) made with clay soil, which were subjected to ultrasound tests with compression wave transducers $(54 \mathrm{kHz})$ and shear $(40 \mathrm{kHz})$. The results indicated that there are directly proportional correlations between the velocities $\left(\mathrm{V}_{\mathrm{P}}\right.$ and $\left.\mathrm{V}_{\mathrm{S}}\right)$ and the apparent density of the cubic specimens of clay soil when the ultrasound tests are performed with the transducers positioned on the side faces of the cubes. However, when the transducers are positioned on the top and bottom of the cubes (direction of compaction), the correlation between density and velocities $\left(V_{P}\right.$ and $\left.V_{S}\right)$ is inversely proportional. This can be justified by the increase in the voids index in the interface region of the compaction layers, which makes the passage of the ultrasonic wave more difficult.

Keywords: rammed earth, compacted soil, non-destructive testing, inspection. 


\section{INTRODUÇÃO}

A história de boa parte dos continentes pode ser contada pela arquitetura, técnica e materiais utilizados em suas construções, as quais muitas são símbolo da parte histórica e cultural de diversos povos. A terra, considerada um dos materiais de construção mais antigos que se tem notícia, foi por muito tempo um dos materiais de construção mais utilizados em edificações (CANIVELL et al., 2018; GALLIPOLI \& MENDES, 2017; NOOR-E-KHUDA \& ALBERMANI, 2019). Assim, tem-se diversas construções tombadas como patrimônio histórico e cultural da humanidade construídas parcial ou totalmente com terra espalhadas por diversos países, como o caso de Portugal e Espanha, além do próprio Brasil, onde o auge da construção com terra se deu no período de colonização. Boa parte das construções no nosso país ainda estão em funcionamento, mesmo após meio século de sua construção.

O uso mais popular desse material é feito compactando-se o material, procedimento base para a viabilização das técnicas de construção como o adobe e a taipa (HOUBEN \& GUILLAUD, 1994). Entretanto, inúmeras são as técnicas de manipulação e aplicação desse material em construções, sendo a diversidade e falta de informações sobre os materiais e procedimentos um dos principais empecilhos dos processos de preservação e restauração dessas construções.

A técnica de ultrassom, utilizada na caracterização, controle e inspeção de materiais de construção, é uma ferramenta validada, entretanto, a o uso desta técnica não destrutiva na caracterização do solo compactado ainda exige estudos específicos e aprofundados. A princípio, as principais contribuições buscadas com a utilização do ultrassom são baseadas na estimativa de parâmetros mecânicos (resistência à compressão e constantes elásticas), as quais requerem estudos para a definição dos procedimentos de ensaio e fatores interferentes.

Algumas pesquisas já identificaram que o pulso ultrassônico, ao passar pelo solo, pode ser influenciado por fatores internos e externos ao material. Pode-se citar alguns exemplos como a metodologia de ensaio(direção de realização das leituras e tipo de transdutor) e os índices físicos da amostra analisada(teor de umidade, densidade, índice de vazios, tipo de solo) (BERNAT-MASO et al., 2017; BUI et al., 2014; CANIVELL et al., 2018; FERREIRA et al., 2013, 2014b; SARRO et al., 2017; SARRO et al., 2015; TEIXEIRA et al., 2015).

Em relação às propriedades mecânicas, diversos autores identificaram a possibilidade de utilização do ultrassom para estimativa de resistência, além da determinação das constantes elásticas de amostras de solo compactado (módulo de elasticidade, cisalhamento e coeficiente de Poisson) (BUI \& MOREL, 2009; CHRIST \& PARK, 2009; HAMMAM \& ELIWA, 2013; HOFFMANN \& GONÇALVES, 2010; INDRARATNA et al., 2012; PADURA et al., 2011; SARRO; \& FERREIRA, 2019; WANG et al., 2006; WANG et al., 2016; ZHEN et al., 2016)

Embora a literatura ainda seja escassa sobre a utilização do ultrassom nesse material, nos últimos anos houve um aumento considerável do interesse da comunidade científica internacional pelo tema, com trabalhos recentes publicados, por exemplo, em Portugal, Irã, Espanha e Austrália (CANIVELL et al., 2018; NOOR-E-KHUDA \& ALBERMANI, 2019; SILVA et al., 2018; TOUFIGH \& KIANFAR, 2019), os quais apontam para a viabilidade de aplicação da técnica.

Buscando consolidar a técnica de ultrassom como uma ferramenta para a caracterização de solos, utilizados tanto em obras geotécnicas como para edificações em terra, o objetivo deste trabalho foi obter as correlações entre os valores da velocidade do pulso ultrassônico, obtidas com ondas de compressão $\left(V_{P}\right)$ e de cisalhamento $\left(V_{S}\right)$, e a direção da transmissão do pulso ultrassônico em corpos de prova cúbicos de solo compactado com diferentes densidades.

Se validada, a técnica de ultrassom em terra compactada pode contribuir durante o processo de caracterização, controle e inspeção de obras de terra compactada, como um meio menos invasivo de investigação.

\section{METODOLOGIA}

\subsection{Material}

Nesta pesquisa utilizou-se uma amostra de solo argiloso, composto por $55 \%$ de argila, $23 \%$ de areia e $22 \%$ de silte, representativa de um dos tipos de solo encontrados no interior do estado de São Paulo, Brasil. Conforme dados obtidos por outros autores, este solo apresenta massa específica dos sólidos de $27,9 \mathrm{kN} / \mathrm{m}$ e baixa plasticidade (ML / A-5), segundo as classificações USCS e HRB, respectivamente (SARRO, 2017; TEIXEIRA, 2014). 


\subsection{Moldagem dos corpos de prova}

A moldagem dos corpos de prova cúbicos foi realizada conforme parâmetros de umidade ótima (WóT) e massa específica seca máxima $\left(\gamma_{d}\right)$ obtidos em ensaios de compactação Proctor, segundo a NBR 7182 (ABNT, 2016). As curvas de compactação (Figura 1) e respectivos valores de $\mathrm{W}_{\text {о̆т e }} \gamma_{d}$ (Tabela 1) foram obtidos anteriormente, através de pesquisas vinculadas ao mesmo grupo de pesquisa do presente estudo (SARRO, 2017; TEIXEIRA, 2014).

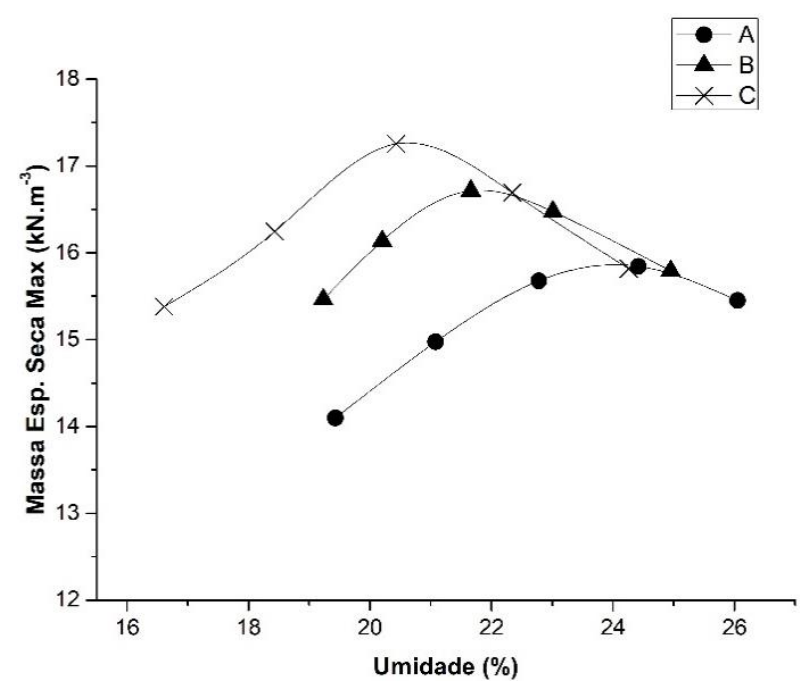

Figura 1: Curvas de compactação das condições adotadas

Confeccionou-se corpos de prova cúbicos de $150 \mathrm{~mm}$ de aresta (Figura 2), utilizando os moldes adotados na BS EN 12390-1 (BS, 2012), adaptados para o material solo. Isto envolveu a confecção de um colar metálico para aumentar a altura útil da fôrma, necessária para a compactação das duas últimas camadas de solo (Figura 3). A definição do formato do corpo de prova (cúbico) foi em função da necessidade de se estabelecer as mesmas dimensões às faces, onde serão posicionados os transdutores durante os ensaios de ultrassom. Outros autores também fizeram uso de formas iguais ou semelhantes para a confecção de corpos de prova de solo compactado (CIANCIO \& GIBBINGS, 2012; FERREIRA et al., 2014a; KARIYAWASAM \& JAYASINGHE, 2016; MICCOLI et al., 2014; MILANI \& LABAKI, 2012; SARRO $e t$ al., 2015).

A compactação foi realizada controlando-se as alturas das camadas, seguindo os resultados obtidos na fase de caracterização física. A realização da compactação com controle de altura e não por controle de golpes, conforme descrito na NBR 7182 (ABNT, 2016), foi adotada em virtude da geometria escolhida para os corpos de prova, que requer um compactador com seção que consiga aplicar carga adequadamente aos cantos da forma. Assim, optou-se por um compactador manual, adaptado, cuja seção de aplicação de carga possui as dimensões internas da forma.

A compactação foi realizada em cinco camadas, para garantir que estas não seriam compactadas apenas superficialmente, tendo em vista que, conforme analisado por Crispim et al., (2015), quanto menor o número de camadas, maior a heterogeneidade do grau de compactação ao longo da altura desses corpos. 


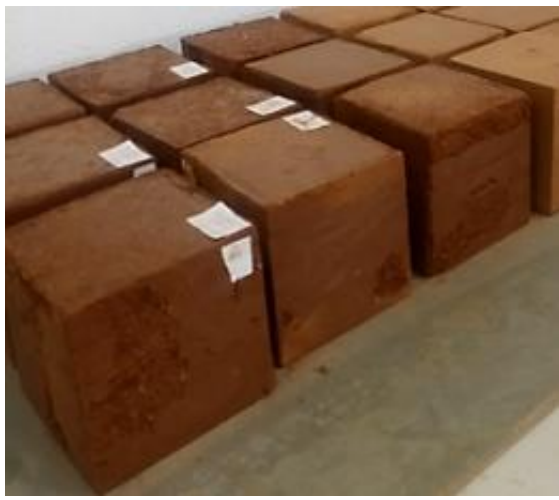

Figura 2: Corpos de prova cúbicos moldados com dois tipos de solo

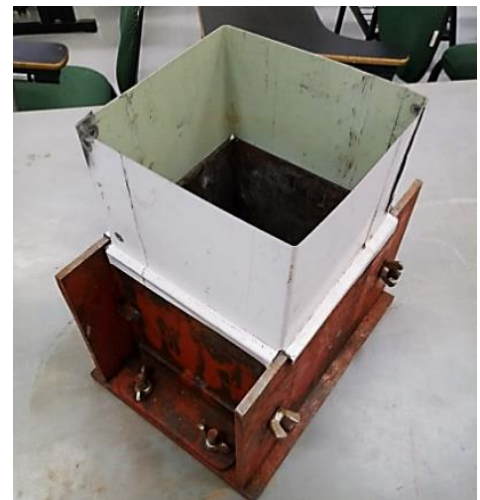

Figura 3: Molde cúbico (BS EN 12390-1, 2012) e colarinho adaptado

\subsection{Ensaio de Ultrassom}

Para a realização das leituras com o ultrassom foram consideradas as direções de leitura da Figura 4, utilizada por Gonçalves et al. (2011) em um estudo sobre ultrassom em concreto. Nesta etapa utilizou-se o equipamento Pundit Lab+ (Proceq, Suíça) (Figura 5 e 6), com transdutores de faces planas e exponenciais, de $54 \mathrm{kHz}$ e $40 \mathrm{kHz}$ de frequência, respectivamente. Assim foi possível obter os valores do tempo de propagação de ondas longitudinais (compressão, pwave) e transversais (cisalhamento, s-wave) e, consequentemente, calcular a velocidade, conforme Equação 1. Os ensaios foram realizados 120 dias após a confecção dos corpos de prova, os quais ficaram expostos às variações de umidade e temperatura do ambiente interno do Laboratório de Solos e Ensaios não destrutivos (LENDs) da FT-UNICAMP.

Onde:

$$
V=\frac{S}{t} \times 10^{6}
$$

$\mathrm{S}=$ Espaço percorrido $(\mathrm{mm})$

$\mathrm{t}=$ tempo $(\mu \mathrm{s})$

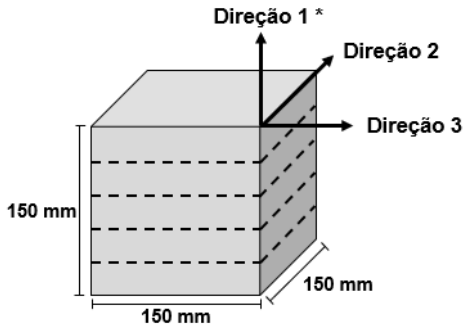

*Direção de compactação das camadas

Figura 4: Eixos de leitura das velocidades

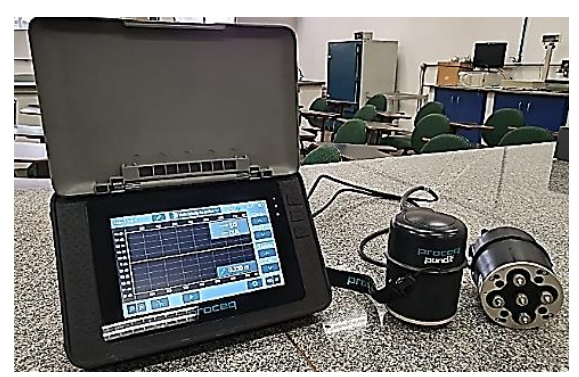

Figura 5: Equipamento de ultrassom Pudit Lab(+)

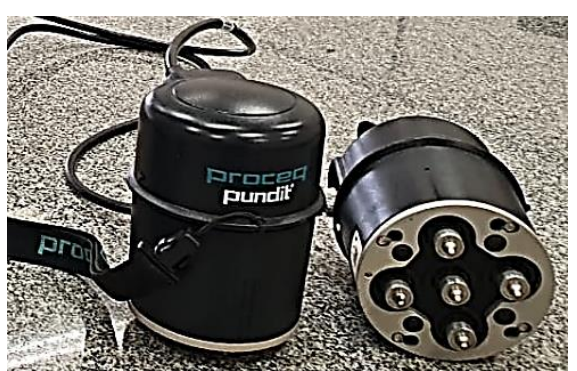

Figura 6: Detalhamento do transdutor exponencial de cisalhamento de 40 $\mathrm{kHz}$ de frequência

Para a caracterização e controle tecnológico de construções em terra, apenas a velocidade longitudinal $\left(\mathrm{V}_{\mathrm{P}}\right)$ seria suficiente, conforme conclusões de outros autores para o mesmo tipo de material (SARRO et al., 2017; SARRO et al., 2015; SARRO \& FERREIRA, 2019). Entretanto, o cálculo das constantes elásticas (Módulo de Elasticidade, Módulo de Cisalhamento e Coeficiente de Poisson), inclui a velocidade obtida com ondas de cisalhamento (Vs). Assim, foi necessário determinar tanto $V_{P}$ quanto $V_{S}$, das condições $A$, B e $C$, tendo em vista que o comportamento pode variar com o tipo de onda. Essas velocidades foram correlacionadas com as respectivas densidades aparentes $\left(\gamma_{w-120}\right)$ determinadas após 120 dias (Tabela 1). 
Tabela 1 - Condições de moldagem dos corpos de prova com diferentes densidades (A, B e C)

\begin{tabular}{c|c|c|c|c|c|c}
\hline \multirow{2}{*}{ Condição } & \multicolumn{3}{|c|}{ Moldagem } & \multicolumn{3}{c}{ Após 120 dias } \\
\cline { 2 - 7 } & $\begin{array}{c}\mathrm{W}_{\text {Oт }} \\
(\%)\end{array}$ & $\begin{array}{c}\gamma_{w} \\
\left(\mathrm{kN} \cdot \mathrm{m}^{-3}\right)\end{array}$ & $\begin{array}{c}\gamma_{d \text { máx }} \\
\left(\mathrm{kN} \cdot \mathrm{m}^{-3}\right)\end{array}$ & $\begin{array}{c}\mathrm{W}_{120} \\
(\%)\end{array}$ & $\begin{array}{c}\gamma_{w-120} \\
\left(\mathrm{kN} \cdot \mathrm{m}^{-3}\right)\end{array}$ & $\begin{array}{c}\gamma_{d-120} \\
\left(\mathrm{kN} \cdot \mathrm{m}^{-3}\right)\end{array}$ \\
\hline $\mathrm{A}$ & 23,4 & 19,6 & 15,9 & 19,3 & 16,4 & 13,8 \\
$\mathrm{~B}$ & 21,9 & 20,3 & 16,7 & 18,2 & 17,1 & 14,5 \\
$\mathrm{C}$ & 20,7 & 21,0 & 17,4 & 17,3 & 17,8 & 15,1 \\
\hline
\end{tabular}

\section{RESULTADOS E DICUSSÕES}

Segundo a comparação das médias de $V_{P}$ (Figura 7), as diferenças entre as diferentes condições de moldagem (A, B e C) não são estatisticamente significativas ( $\mathrm{p}$-valor $>0,05$ ). Os valores médios de $\mathrm{V}_{\mathrm{P}}$ para as condições de moldagem deste trabalho foram da mesma ordem de grandeza que os obtidos por Sarro \& Ferreira (2019) para corpos de prova cilíndricos, ensaiados no sentido das camadas de compactação e expostos em ambiente laboratorial por 120 dias. Os valores de $\mathrm{V}_{\mathrm{P}}$ obtidos por esses autores, para amostras moldadas na umidade ótima, foi de aproximadamente $1400 \mathrm{~m} . \mathrm{s}^{-1}$. Considerando a mesma direção de leitura, no sentido das camadas de compactação (direção 1), neste trabalho os valores médios de $V_{P}$ foram de $1550 \mathrm{~m} \cdot \mathrm{s}^{-1}$ (Figura 8).

Bernat-Maso et al. (2017) também investigaram a eficiência da técnica de ultrassom em corpos de prova cúbicos, utilizando transdutores longitudinais de $55 \mathrm{kHz}$ de frequência. Os autores expuseram as amostras a ambiente laboratorial, por aproximadamente 60 dias, verificando a diferença da velocidade medida no topo, meio e base dos corpos de prova. Para as leituras realizadas no topo, onde os corpos de prova estavam com menor teor de umidade, a $V_{\mathrm{P}}$ obtida foi de aproximadamente $1200 \mathrm{~m} \cdot \mathrm{s}^{-1}$.

Se comparadas as direções de leitura 1, 2 e 3, as velocidades longitudinais também não são diferentes estatisticamente para nenhuma delas (Figura 8). Na literatura, em painéis monolíticos de solo-cimento, também foram verificadas as velocidades considerando as diferentes direções de leitura, sendo estas correspondentes a comprimento, altura e largura (FERREIRA et al., 2014a). Os autores em questão também verificaram que não houve diferença estatística entre as direções consideradas. Apesar disso, para a direção onde a onda se propaga por todas as camadas, correspondente a direção 1, a velocidade foi menor do que nas demais direções, sendo esse comportamento diferente do observado neste trabalho (Figura 8). Canivell et al. (2018) também analisaram a variação da velocidade em função do teor de umidade, densidade e direção de realização de leitura de cubos de solo compactado e obtiveram menores velocidades para as amostras moldadas próxima a umidade ótima e medidas no sentido de compactação.

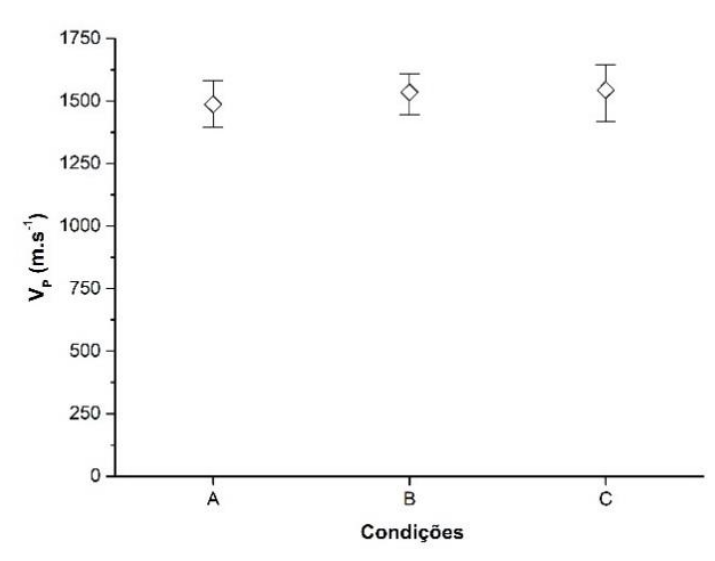

Figura 7: Comparação geral da $V_{P}$ por condição de moldagem dos corpos de prova cúbicos $(\mathrm{A}, \mathrm{B}$ e $\mathrm{C})$.

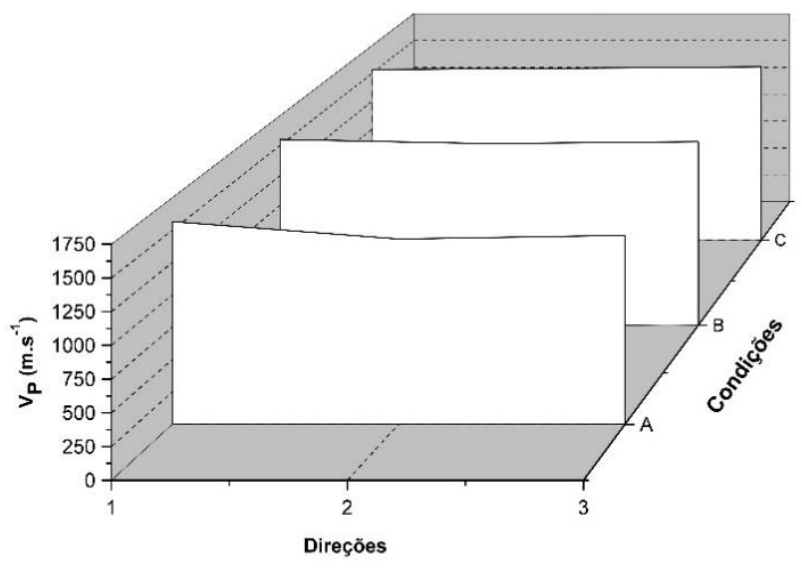

Figura 8: Comparação da $V_{P}$ média por direção de transmissão do pulso ultrassônico e condições de moldagem dos cubos (A, B e C)

Segundo a comparação das médias de $\mathrm{V}_{\mathrm{S}}$ (Figura 9), as diferenças entre as condições de moldagem consideradas nesse trabalho (A, B e C) não são estatisticamente significativas ( $p$-valor > 0,05). Esses valores foram da mesma ordem de 
grandeza que os obtidos por Sarro \& Ferreira (2019) para corpos de prova cilíndricos, ensaiados no sentido das camadas de compactação e expostos em ambiente laboratorial por 120 dias. Os valores de $\mathrm{V}_{\mathrm{S}}$ obtidos por esses autores, para amostras moldadas na umidade ótima, foi de aproximadamente $900 \mathrm{~m} . \mathrm{s}^{-1}$.

Se comparadas as direções de leitura 1,2 e 3, as velocidades transversais também não são diferentes estatisticamente para nenhuma delas (Figura 10). Considerando a mesma direção de leitura, no sentido das camadas de compactação (direção 1), neste trabalho os valores médios de $\mathrm{V}_{\mathrm{S}}$ foram de $946 \mathrm{~m} \cdot \mathrm{s}^{-1}$.

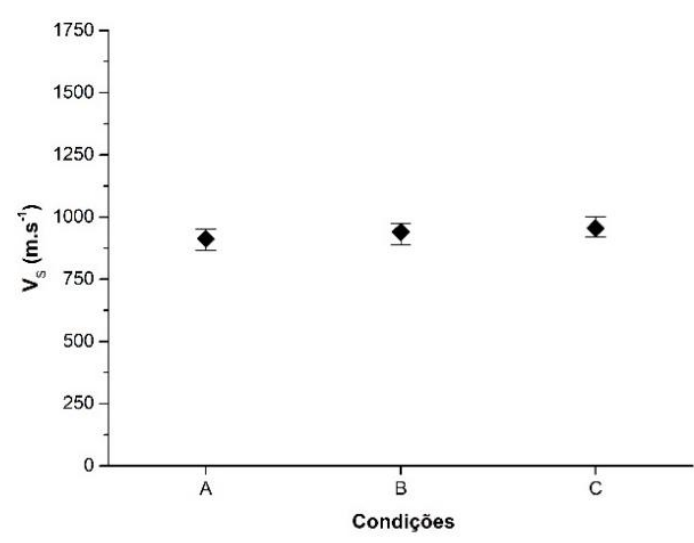

Figura 9: Comparação de $\mathrm{V}_{\mathrm{S}}$ por condição de moldagem

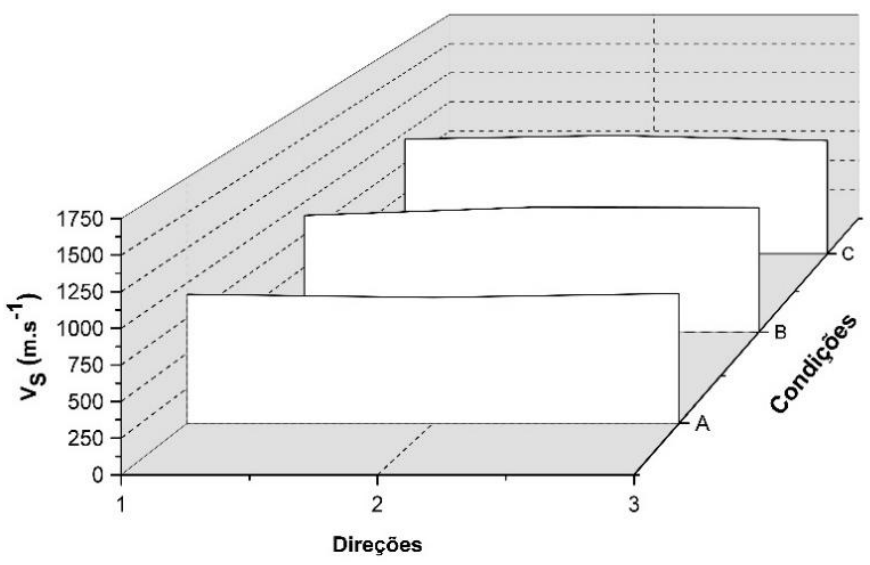

Figura 10: Comparação da $\mathrm{V}_{\mathrm{S}}$ média por direção de realização de leitura

As $\mathrm{V}_{\mathrm{P}}$ e $\mathrm{V}_{\mathrm{S}}$ foram ainda correlacionadas com as respectivas densidades aparentes, determinadas após os 120 dias, instantes antes da realização do ensaio de ultrassom. Quando analisadas de forma geral, desconsiderando que haja diferença entre as direções de leitura, não houve correlação entre essas duas variáveis, nem no que diz respeito a $V_{P}(R=0,55)$ nem relacionado a $\mathrm{V}_{\mathrm{S}}(0,71)$ (Figura 11 e Tabela 2). Entretanto, as velocidades e respectivas massas específicas dos corpos de prova, se correlacionadas considerando as direções de forma individual, indicaram linearidade do comportamento entre as variáveis para as direções 2 e 3, tanto para $V_{P}\left(R=0,96\right.$ e $R=0,97$ respectivamente), quanto para $V_{S}(R=0,94$ e $R=1,00$, respectivamente). Já para a direção 1 , o coeficiente de correlação foi inversamente proporcional para $V_{P}(R=-0,99)$ e insatisfatório para $\mathrm{V}_{\mathrm{S}}(\mathrm{R}=0,59)$.

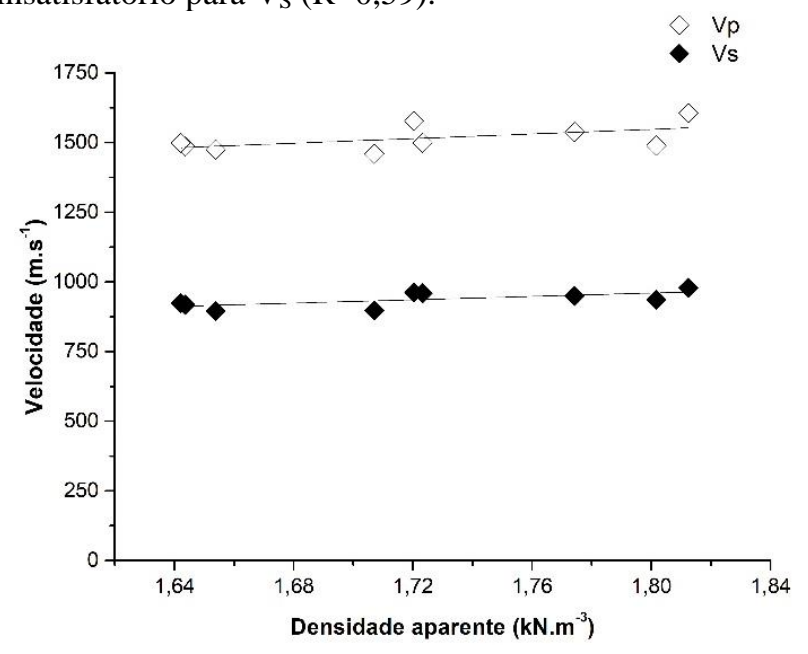

Tabela 2 - Correlação entre os valores de velocidade, obtidos com ondas de compressão (Vp) e ondas de cisalhamento (Vs) com a densidade aparente dos cubos

Figura 11: Gráfico de correlação entre os valores de velocidade com a densidade aparente dos cubos

\begin{tabular}{ccc}
\hline & $\mathrm{V}_{\mathrm{P}}$ & $\mathrm{V}_{\mathrm{S}}$ \\
\hline $\mathrm{R}^{2}$ & 0,31 & 0,46 \\
$\mathrm{R}$ & 0,55 & 0,71 \\
Eq. & $y=414,49 x+801,79$ & $y=418,85 x+300,84$ \\
\hline
\end{tabular}

Chagas et al. (2018) e Indraratna et al. (2012), obtiveram valores de $\mathrm{V}_{\mathrm{S}}$ menores dos que os obtidos nessa pesquisa, tendo em vista que outra técnica foi utilizada para obter a velocidade de cisalhamento (Bender Elements), entretanto, os autores observaram um comportamento da relação entre velocidade e massa especifica semelhante a curva de compactação, sendo a velocidade diretamente proporcional a densidade de ramo úmido, até o teor ótimo e inversamente proporcional após esse ponto. Por outro lado, Yang et al. (2012), que analisou diferentes materiais com umidade e densidade nas condições ótimas, indicou que a $\mathrm{V}_{\mathrm{S}}$ é diretamente proporcional a densidade. 


\section{CONLUSÕES}

O ultrassom foi capaz de identificar as diferenças entre as diferentes condições de moldagem, dependendo da direção de realização do ensaio. Foram obtidos valores de correlação satisfatórios com a densidade aparente ao utilizar os valores de velocidade nas direções 2 e 3 , que correspondem a largura e comprimento da peça.

Quando realizadas leituras de na direção 1, no sentido de compactação do corpo de prova, os valores de correlação da velocidade com a densidade apresentaram diferença de comportamento, sendo $V_{P}$ inversamente proporcional e $V_{S}$ com um coeficiente de correlação muito abaixo do demais. Essa diferença considerando a direção 1 ocorreu mesmo a velocidade não tendo apresentado diferença estatística com as obtidas para as direções 2 e 3. Em ambos os casos, as velocidades foram afetadas pela presença das camadas de compactação, entretanto, $\mathrm{V}_{\mathrm{S}}$ foi mais afetada, pela própria natureza de propagação que esta exerce, sendo o movimento perpendicular a direção de propagação.

Esse comportamento indica que a técnica pode ser utilizada na caracterização de construções em terra, desde que as leituras sejam realizadas considerando a largura ou comprimento da estrutura. Tendo em vista que essas são de fato as direções passiveis de acesso durante uma inspeção, a técnica demonstra viabilidade de aplicação para os fins propostos.

\section{REFERÊNCIAS}

ABNT. NBR 7182: Solo - Ensaio de Compactação. Rio de Janeiro, 2016.

BERNAT-MASO, E. et al. Ultrasound transmission method to assess raw earthen materials. Construction and Building Materials, v. 156, p. 555-564, 2017.

BS. BS EN 12390-1: Testing hardened concrete - Shape, dimensions and other requirements for specimens and mouldsBrusselsBritish Standard, , 2012.

BUI, Q.-B.; MOREL, J.-C. Assessing the anisotropy of rammed earth. Construction and Building Materials, v. 23, n. 9, p. 3005-3011, 2009.

BUI, Q. B. et al. Effect of moisture content on the mechanical characteristics of rammed earth. Construction and Building Materials, v. 54, p. 163-169, 2014.

CANIVELL, J. et al. Considerations on the physical and mechanical properties of lime-stabilized rammed earth walls and their evaluation by ultrasonic pulse velocity testing. Construction and Building Materials, v. 191, p. 826-836, dez. 2018.

CHAGAS, B. B. N. DAS; CAVAlLARO, F. DE A.; MARINHO, F. A. M. Análise da influência da umidade na variação da velocidade de propagação de ondas de cisalhamento em solos compactados utilizando Bender Elements. (ABMS, Ed.)XIX Congresso Brasileiro de Mecânica dos Solos e Engenharia Geoténica. Anais...Salvador, Brasil: 2018

CHRIST, M.; PARK, J. B. Ultrasonic technique as tool for determining physical and mechanical properties of frozen soils. Cold Regions Science and Technology, v. 58, n. 3, p. 136-142, 2009.

CIANCIO, D.; GIBBINGS, J. Experimental investigation on the compressive strength of cored and molded cementstabilized rammed earth samples. Construction and Building Materials, v. 28, n. 1, p. 294-304, 2012.

CRISPIM, F. A. et al. Compactação de solos em laboratório: Efeito do diâmetro e do número de camadas do corpo de prova. Revista Árvore, v. 39, n. 3, p. 535-542, 2015.

FERREIRA, G. C. DOS S. et al. Determinação de propriedades de solo compactado utilizando ensaios de ultrassom. $12^{\mathrm{a}}$ Conferência sobre Tecnologia de Equipamentos. Anais...Porto de Galinhas, PE: Abendi, 2013

FERREIRA, G. C. DOS S. et al. Influência das camadas de compactação em inspeções de painéis monolíticos de solo-cimento por ultrassom. (Abendi, Ed.)ConaEnd\&Iev. Anais...São Paulo, SP: 2014a 
FERREIRA, G. C. DOS S. et al. Influência das camadas de compactação em inspeções de painéis monolíticos de solo-cimento por ultrassom. Congresso Nacional de Ensaios Não Destrutivos e Inspeção - ConaEnd2014. Anais...São Paulo, SP: Abendi, 2014b

GALLIPOLI, D.; MENDES, J. A geotechnical perspective of raw earth building. p. 463-478, 2017.

GONÇALVES, R.; GIACON JUNIOR, M.; LOPES, I. M. Determining the concrete stiffness matrix through ultrasonic testing. ENGENHARIA AGRÍCOLA, v. 31, n. 3, p. 427-437, 2011.

HAMMAM, A. H.; ELIWA, M. Comparison between results of dynamic \& static moduli of soil determined by different methods. HBRC Journal, v. 9, n. 2, p. 144-149, 2013.

HOFFMANN, M. V; GONÇALVES, R. Análise da qualidade da taipa de pilão por meio de ondas ultra- sônicas. Terra Brasil 2010 - III Congresso de Arquitetura e construção com terra no Brasil. Anais...Campo Grande - MS: 2010

HOUBEN, H.; GUILLAUD, H. Soil suitability, Construction Methods and Production Methods. In: Earth construction: a comprehensive guide. Intermediate Technology Publications, 1994.

INDRARATNA, B.; HEITOR, A.; RUJIKIATKAMJORN, C. Effect of compaction energy on shear wave velocity of dynamically compacted silty sand soil. 5th Asia-Pacific Conference on Unsaturated Soils. Anais...2012

KARIYAWASAM, K. K. G. K. D.; JAYASINGHE, C. Cement stabilized rammed earth as a sustainable construction material. Construction and Building Materials, v. 105, p. 519-527, 2016.

MICCOLI, L.; MÜLLER, U.; FONTANA, P. Mechanical behaviour of earthen materials: A comparison between earth block masonry, rammed earth and cob. Construction and Building Materials, v. 61, p. 327-339, 2014.

MILANI, A. P. S.; LABAKI, L. C. Physical, Mechanical , and Thermal Performance of Cement-Stabilized Rammed Earth - Rice Husk Ash Walls. Journal of Materials in Civil Engineering, v. 24, n. 6, p. 775-782, 2012.

NOOR-E-KHUDA, S.; ALBERMANI, F. Mechanical properties of clay masonry units : Destructive and ultrasonic testing. Construction and Building Materials, v. 219, p. 111-120, 2019.

PADURA, A. B.; SEVILLA, J. B.; NAVARRO, J. G. Bearing capacity diagnosis of Santiago church (Jerez de la Frontera, Spain). Construction and Building Materials, v. 25, n. 5, p. 2519-2527, 2011.

SARRO, W. DE S. et al. Influência dos transdutores no ensaio de ultrassom aplicado a solo compactado. (Abendi, Ed.) $14^{\text {a }}$ Conferência sobre tecnlogia de equipamentos. Anais...Rio de Janeiro: Abendi, 2017

SARRO, W. DE S. Constantes elásticas de solo compactado a partir do ensaio de ultrassom. [s.1.] Universidade Estadual de Campinas, 2017.

SARRO, W. DE S.; FERREIRA, G. C. DOS S.; GALLETTO, A. Técnica de ultrassom aplicada na inspeção de edificações construídas em solo compactado. (IBRACON, Ed.)Anais do $57^{\circ}$ Congresso Brasileiro do Concreto CBC2015. Anais...Bonito, MS: 2015

SARRO, W. S.; FERREIRA, G. C. S. Soil Elastic Modulus Determined by Ultrasound Tests. Soils and Rocks, v. 42 , n. 2, p. 117-126, 2019.

SILVA, R. A. et al. Evaluating the seismic behaviour of rammed earth buildings from Portugal : From simple tools to advanced approaches. Engineering Structures, v. 157, n. June 2017, p. 144-156, 2018.

TEIXEIRA, I. Estabilização de um solo laterítico argiloso para utilização como camada de pavimento. [s.l.] Universidade Estadual de Campinas - UNICAMP, 2014.

TEIXEIRA, I. et al. Influência da granulometria e umidade nas propriedades de solos a partir de ensaios destrutivos e não destrutivos. XXIX Congresso Nacional de Pesquisa em Transporte da ANPET. Anais...Ouro Preto, MG: ANPET, 


\section{CBPAT 2020 \\ CONGRESSO BRASILEIRO DE PATOLOGIA DAS CONSTRUÇÕES \\ DE 15 A 17 DE ABRIL | FORTALEZA - CE \\ ISBN 978-65-86819-05-2}

2015

TOUFIGH, V.; KIANFAR, E. The effects of stabilizers on the thermal and the mechanical properties of rammed earth at various humidities and their environmental impacts. Construction and Building Materials, v. 200, p. 616-629, 2019.

WANG, D. Y. et al. Application of ultrasonic technology for physical-mechanical properties of frozen soils. Cold Regions Science and Technology, v. 44, n. 1, p. 12-19, 2006.

WANG, Y.; LI, X.; ZHENG, B. Experimental study on mechanical properties of clay soil under compression by ultrasonic test. European Journal of Environmental and Civil Engineering, v. 8189, n. June, 2016.

YANG, C.; CAI, W.; DOU, H. Research on comparison of the maximum dynamic shear modulus test. Procedia Engineering, v. 28, n. 2011, p. 230-234, 2012.

ZHEN, H.; HE-LIN, F.; JIA-BING, Z. Relationship between Wave Velocity and Strength of Compacted Expansive Soil , and Application of Acoustic Technology. EJGE, p. 2115-2126, 2016. 